Ann. Génét. Sél. Anim. I978, 10 (I), 29-45.

\title{
Analyse et décomposition de la variabilité intergénotypique de la consommation alimentaire pour des jeunes bovins
}

\author{
J. J. COLLEAU
}

avec la collaboration technique de M. Dupont, D. Tanguy et J. J. Chambeyron

\author{
Station de Génétique quantitative et appliquée, I.N.R.A., \\ Centre national de Recherches zootechniques \\ 78350 Jouy-en-Josas, France
}

\begin{abstract}
Résumé
Les consommations alimentaires pendant la période d'engraissement (de 10 à 16 mois d'âge) sont mesurées sur $17 \mathrm{r}$ taurillons appartenant à 7 types génétiques distincts : trois races pures (Holstein, Normand, Charolais) et quatre croisements divers entre ces races pures.

Elles sont analysées en fonction des facteurs type génétique et année d'engraissement et des trois variables suivantes : poids moyen pendant l'engraissement lié aux besoins d'entretien, quantités de lipides et de protéines déposées, liées à l'énergie fixée pendant l'engraissement. Chacune de ces trois variables est en relation significative avec la consommation indépendamment des deux autres. Le coefficient de régression partielle de la consommation totale sur la quantité de lipides déposés est égal à $72,2( \pm 5,5) \mathrm{KJ}$ Énergie Métabolisable/g de lipides. Celui qui correspond aux protéines déposées est égal à $7 \mathrm{I}, 7( \pm \mathrm{I} 4,8) \mathrm{KJ} / \mathrm{g}$. Le cœefficient de régression partielle sur le poids est également significatif, cependant un ajustement simultané des consommations pour chacune des variables laisse subsister encore des différences significatives entre types génétiques. Pour expliquer ce résultat, on avance l'hypothèse de l'hétérogénéité du coefficient de régression partielle sur le poids, les coefficients étant plus élevés pour les animaux de type laitier (Holstein) que pour ceux de type à viande (Charolais). Le coefficient de proportionnalité au poids métabolique $\left(p^{0,75}\right)$ est de $\mathrm{I}_{4} \mathrm{I}( \pm 24) \mathrm{Kcal} \mathrm{EM} / \mathrm{KG}^{0,75}$ sur l'ensemble des animaux. Il serait de $\mathrm{I}^{2}$ pour les taurillons Holsteins et de $\mathrm{r}_{32}$ pour les taurillons Charolais.

La variation intergénotypique pour l'efficacité alimentaire ( $\mathrm{Kg}$ MS consommés $/ \mathrm{Kg}$ de gain)

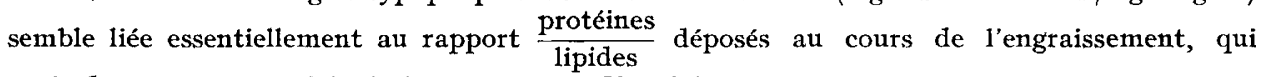
varie de 0,54 en race $H o l s t e i n$ à $\mathrm{I}$, or en race Charolaise.
\end{abstract}

\section{Introduction}

Les races bovines mixtes et les races laitières spécialisées présentent par rapport aux races spécialisées à viande, les races continentales du moins, le désavantage d'une moindre efficacité alimentaire pour la production de viande, alors que dans 
ce cas, le coût de l'alimentation représente une forte proportion du coût total de production.

Nous avons tenté antérieurement de préciser les différences qui pouvaient exister entre grands types de bovins en faisant état des premiers résultats d'une expérimentation où l'on comparait les races Holstein (spécialisée laitière), Normande (mixte) et Charolaise (spécialisée viande) et leurs croisements (COLLEAU, I974, I975). De grandes différences d'efficacité alimentaire étaient apparues mais les raisons possibles d'une telle variation entre races n'étaient pas clairement explicitées. Nous nous proposons dans cet article d'aborder ce point en nous basant sur un échantillon plus important d'animaux issus de cette expérimentation.

Les causes de variation de l'efficacité alimentaire sont théoriquement nombreuses. Elles peuvent porter :

a) sur les besoins d'entretien, qui représentent plus de la moitié des besoins totaux en énergie métabolisable;

b) sur le niveau de la vitesse de croissance;

c) sur la composition du croît en eau, protéines et lipides, qui détermine la quantité d'énergie fixée;

d) sur le rendement de l'énergie métabolisable pour la fixation de protéines et de lipides.

La connaissance de la composition du croît constitue sans doute le minimum à obtenir pour une analyse génétique du problème. Depuis plusieurs années, on dispose en France d'équations d'estimation de la composition de la carcasse de jeunes bovins en muscle, os et gras séparables à la dissection (GEAY et BERANGER, I969). Récemment, RoBELIN et GEAY (I975) ont proposé de nouvelles équations d'estimation à partir d'un matériel animal plus abondant. Ils ont aussi montré, ce qui est d'un très grand intérêt pour nous, que ces équations étaient indépendantes du type génétique de l'animal (sauf dans une certaine mesure pour la prédiction du poids du squelette). Les mêmes auteurs viennent d'étendre leurs travaux à l'estimation des quantités de lipides et de protéines présentes dans l'animal entier (ROBELIN et GEAY, I978), les coefficients d'estimation ne dépendant pas, là encore, du type génétique. L'intérêt de la prédiction de telles variables est évident pour toute étude portant sur l'efficacité alimentaire, c'est pourquoi nous en tiendrons compte dans la présente analyse.

\section{Matériel et méthodes}

\section{a) Matériel animal}

Il est constitué de I7I taurillons issus de l'expérimentation bovine du Pinau-Haras. Ils se répartissent sur 4 années de naissance $(69-70,70-7 \mathrm{I}, 72-73,74-75)$ et appartiennent à 7 types génétiques :

- Holstein (HF) 32 animaux.

- $3 / 4$ Holstein I/4 Normand (3/4 HF I/4 NO) I9 animaux.

- Holstein $\times$ Normand $(\mathrm{HF} \times \mathrm{NO}) 30$ animaux.

- Normand (NO) 59 animaux.

- Charolais $\times$ Normand $(\mathrm{CH} \times \mathrm{NO})$ I5 animaux.

- Charolais (CH) 6 animaux.

- Holstein $\times$ Charolais $(\mathrm{HF} \times \mathrm{CH})$ Io animaux. 
Jusqu'à Io mois, ces taurillons sont élevés au domaine du Pin-au-Haras (Orne). L'allaitement artificiel durait trois mois les deux premières années et deux mois les deux dernières années. La périođe d'élevage se déroule ensuite jusqu'à 9 mois avec distribution de foin et de concentré, ou d'herbe et de concentré suivant la saison, la croissance moyenne approchant I $\mathrm{kg} /$ jour; la consommation n'est pas alors mesurée. A ro mois, après une transition alimentaire d'un mois, les animaux sont transférés à l'étable d'engraissement de la Minière (Yvelines) où ils reçoivent ad libitum pendant une durée fixe de $\mathrm{I} 82$ jours un aliment déshydraté comprenant $70 \mathrm{p}$. Ioo de luzerne à I $6 \mathrm{p}$. IOo de matières azotées totales et $30 \mathrm{p}$. IOO de pulpe de betterave. La teneur en énergie métabolisable de la luzerne peut être estimée à $2060 \mathrm{Kcal} / \mathrm{kg}$ MS et celle de la pulpe à $2750 \mathrm{Kc} c 1 / \mathrm{kg}$ MS (DEMARQuILLY et al., I978). La teneur moyenne pour la ration s'élève ainsi à $2270 \mathrm{Kcal} / \mathrm{kg} \mathrm{MS}$. Pendant la période d'engraissement, les quantités d'aliment ingérées sont contrôlées 5 jours sur 7 (du lundi au vendredi).

\section{b) Variables}

Les variables étudiées sont les suivantes :

I) La consommation moyenne journalière de matière sèche pendant l'engraissement.

2) 3) 4) Les poids initiaux, moyens et finaux au cours de l'engraissement.

5) Le poids vif vide à l'abattage.

6) 7) 8) Les poids de muscle, de gras et d'os dans la carcasse calculés suivant les formules de RoBELIN et GEAy (I975) qui prennent en considération le poids de carcasse chaude, les poids de muscle, de gras et d'os fournis par la dissection de la $\mathrm{II}^{\mathrm{e}}$ côte, le poids des dépôts adipeux périrénaux et précruraux, le poids des os canons, le rapport (épaisseur cuisse/longueur cuisse). $\mathbf{R}^{2}=0,96$ à 0,99 sur l'échantillon étudié par les auteurs.

9) Le poids du $5^{\mathrm{e}}$ quartier sans le gras, calculé de la manière suivante : poids vif vide-poids de carcasse-(poids de gras de rognon + poids de gras de mésentère + poids de gras de péritoine + poids de gras de cœur), l'expression entre parenthèses constituant ce que nous appelons le poids de gras du $5^{\mathrm{e}}$ quartier.

Io) I,e poids de masse " maigre " égal à la somme du poids de muscle dans la carcasse et du poids du $5^{\mathrm{e}}$ quartier sans le gras. En fait, intervient dans cette masse "maigre " une faible proportion d'os apportée par la tête et les pieds. Cette somme n'est pas prise en considération arbitrairement : en effet, les travaux de Schulz et al. (I974) sur taurillons Frisons montrent que les teneurs en protéines et en lipides des deux éléments de la somme sont voisines. Si l'on prend par exemple, les valeurs trouvées pour un poids vif vide moyen de $509 \mathrm{~kg}$, poids voisin de celui de nos taurillons, on trouve que les muscles squelettiques sont constitués de $20,4 \mathrm{p}$. Ioo de protéines et de 4,7 p. Ioo de lipides. Les valeurs trouvées pour le $5^{\mathrm{e}}$ quartier en excluant les morceaux de gras séparables à la dissection, sont alors de $2 \mathrm{I}, 8 \mathrm{p}$. Ioo pour les protéines et de $6,2 \mathrm{p}$. Ioo pour les lipides.

I I) Le poids des morceaux gras du $5^{\mathrm{e}}$ quartier, défini plus haut.

I2) Le poids des dépôts adipeux totaux (D) égal à la somme du poids de gras dans la carcasse et du poids de gras dans le $5^{\mathrm{e}}$ quartier. 
I3) Le poids total de lipides (carcasse $+5^{\mathrm{e}}$ quartier) estimé d'après les travaux de Robein et Geay (I978), lipides $(\mathrm{kg})=\mathrm{I}, 40 \mathrm{I} 3 \mathrm{D}^{0,9387}$ (coefficient de variation résiduel $=4,9 \mathrm{p}$. IOO).

I4) Le poids total de protéines (carcasse $+5^{\text {e }}$ quartier) estimé d'après les mêmes auteurs,

protéines $(\mathrm{kg})=0, \mathrm{I} 227 \mathrm{MD}^{1,1001}$ (coefficient de variation résiduel $=2,8 \mathrm{p}$. I00). avec $\mathrm{MD}=$ Masse délipidée $=$ Poids vif vide - Poids de lipides.

I5) Le poids de lipides déposés au cours de l'engraissement.

I6) Le poids de protéines déposées au cours de l'engraissement.

Les deux dernières variables ont été calculées par différence entre les quantités présentes à la fin et au début de l'engraissement. Les quantités correspondant au début de l'engraissement ont été établies d'après les résultats de ScHulz (I974) sur mâles Frisons aux environs de 3 IO $\mathrm{kg}$ de poids vif (lipides et protéines = respectivement 8 p. Ioo et $I_{5}, 8$ p. Ioo du poids vif).

\section{c) Méthodes statistiques}

L'ensemble des variables a été analysé suivant le modèle

$$
\mathrm{Y}_{i j k}=\mu+\alpha_{i}+\beta_{j}+\varepsilon_{i j k}
$$

$\mathrm{Y}_{i j k}=$ observation sur le $k^{\mathrm{e}}$ animal du $i \mathrm{e}$ type génétique la $j^{\mathrm{e}}$ année.

$\mu=$ une constante commune à toutes les observations.

$\alpha_{i}=$ effet du $i$ e génotype $(i=\mathrm{I}$ à 7$)$ avec $\Sigma_{i} \hat{\alpha}_{i}=0$.

$\beta_{j}=$ effet de la $j$ e année $(j=\mathrm{I}$ à 4$)$ avec $\Sigma_{j} \widehat{\beta}_{j}=0$.

$\varepsilon_{i j k}=$ erreur aléatoire supposée normale $\left(0, \sigma_{\varepsilon}^{2}\right)$; les $\varepsilon_{i j k}$ sont également supposées indépendantes entre elles.

L'utilisation de la méthode des moindres carrés a permis d'estimer les $\alpha_{i}$ et $\beta_{j}$, de tester leur signification et également de calculer les corrélations résiduelles (corrélations entre les écarts $\hat{\varepsilon}$ au modèle pour un couple de variables.)

La consommation alimentaire a été analysée ensuite suivant le modèle (2) à 2 facteurs et plusieurs covariables :

$$
\mathrm{Y}_{i j k}=\mu+\alpha_{i}+\beta_{j}+\sum_{l} \lambda_{l} \mathrm{X}^{l_{i j k}}+\varepsilon_{i j k}
$$

où $\mathrm{X}^{l_{i j k}}$ est la valeur de la $l^{\mathrm{e}}$ covariable pour l'animal, ijk et $\lambda_{l}$ le coefficient de régression partielle sur la $l$ e covariable. Les mêmes calculs que sur le modèle ( $\mathrm{r}$ ) ont été faits.

Dans ce modèle (2) et dans la mesure où les facteurs sont réellement additifs, les coefficients de régression $\lambda$ ont la signification de coefficient inter-animal intrarace intra-année. Dans la mise en œuvre des calculs, les $\lambda$ ont été supposés ne pas dépendre du type génétique. 


\section{Résultats}

a) Analyse intergénotypique sur l'ensemble des variables (modèle I) $\left(\hat{\mu}+\widehat{\alpha_{i}}\right)$.

Au tableau I figurent les estimations de moyennes par types génétiques

Si l'on compare les races pures, on constate que la consommation est maximale en race Holstein et minimale en race Normande, la race Charolaise se plaçant à un niveau intermédiaire. Ces différences reflètent exactement des différences d'appétit (consommation/poids) $)^{0,75}$ ) déjà notées pour les races Frisonne et Charolaise par JARRIGE et al, (I97I). En dépit du fait que les taurillons Normands sont les plus légers, on note des tendances uniformes quand on va de la race Holstein à la race Charolaise en passant par la race Normande.

- augmentation du poids de muscle dans la carcasse,

- diminution du poids de gras et du poids d'os dans la carcasse,

\section{TABLEAU I.}

Moyennes par type génétique et écarts-type résiduels

Means by genetic type and residual standard deviations

\begin{tabular}{|c|c|c|c|c|c|c|c|c|}
\hline Variable & $\begin{array}{c}\mathrm{HF} \\
\left(u=3^{2}\right)\end{array}$ & $\begin{array}{l}3 / 4 \mathrm{HF} \\
1 / 4 \mathrm{NO} \\
(n=19)\end{array}$ & $\left|\begin{array}{c}\mathrm{HF} \\
\mathrm{NO} \\
(n=3 \mathrm{O})\end{array}\right|$ & $\begin{array}{c}\text { NO } \\
(n=59)\end{array}$ & $\begin{array}{l}\mathrm{CH} \times \\
\mathrm{NO} \\
(n=\mathrm{I} 5)\end{array}$ & $\begin{array}{c}\mathrm{CH} \\
(n=6)\end{array}$ & $\begin{array}{l}\mathrm{HF} \times \\
\mathrm{CH} \\
(n=\mathrm{IO})\end{array}$ & $\begin{array}{l}\text { Écart- } \\
\text { type } \\
\text { résiduel }\end{array}$ \\
\hline I. Consommation MS & & & & & & & & \\
\hline $\mathrm{Kg} / \mathrm{j}$ & Io,44 & 10,07 & 10,22 & 9, I I & 9,65 & 9,66 & 10,16 & 0,97 \\
\hline 2. Poids initial $(\mathrm{kg})$ & 316 & 316 & 326 & 304 & 312 & 314 & 324 & 29,7 \\
\hline 3. Poids moyen $(\mathrm{kg})$ & 437 & 433 & 453 & 423 & $44^{\circ}$ & 458 & $45^{I}$ & 34,7 \\
\hline 4. Poids final $(\mathrm{kg})$. & 545 & $53^{\circ}$ & 560 & $5 z 6$ & 556 & 577 & 560 & $43, \mathrm{I}$ \\
\hline 5. Poids vif vide $(\mathrm{kg})$ & 468 & $45^{8}$ & 487 & 456 & 484 & 504 & 490 & 38,7 \\
\hline 6. Muscle carcasse $(\mathrm{kg})$ & I 84,4 & I 78,5 & 200,5 & I 96,5 & 221,4 & 243,5 & $2 \mathrm{II}, 6$ & 19,75 \\
\hline 7. Gras carcasse $(\mathrm{kg})$ & 54,4 & 53,9 & 58,4 & 48,2 & 50,6 & 47,7 & $5^{2,9}$ & 10,00 \\
\hline 8. Os carcasse $(\mathrm{kg})$. . & 49,0 & 48,7 & 50,8 & 47,9 & $5^{0,2}$ & 47,9 & 49,3 & 3,8 \\
\hline 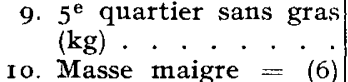 & 147,3 & $\mathrm{r} 45,3$ & 146,2 & 139,0 & 136,7 & 141,6 & 149,8 & II, 4 \\
\hline II. Gras du $5^{\mathrm{e}}$ quartier & $33 \mathrm{I}, 7$ & $3^{2} 3,8$ & 346,7 & 335,5 & $35^{8,1}$ & $3^{8} 5,1$ & $36 r, 4$ & 28,0 \\
\hline 12. $\stackrel{(\mathrm{kg})}{\text { Dépôts }} \cdot \overrightarrow{\text { adipeux }}$ & 28,8 & 27,9 & 26,8 & $20, \mathrm{I}$ & 21,0 & 18,9 & 22,2 & 5,5 \\
\hline taux $(\mathrm{kg})$ & 83,2 & $8 \mathrm{I}, 8$ & 85,2 & 68.3 & 71,6 & 66,6 & $75, \mathrm{I}$ & 14,7 \\
\hline 13. Lipides (kg) & 88,9 & 87,5 & 90,9 & 73,9 & 77,2 & $7^{2,2}$ & 80,8 & I 4,8 \\
\hline 14. Protéines $(\mathrm{kg})$. & 84,3 & 82,2 & 88,4 & 85,0 & $9 \mathrm{I}, \mathrm{I}$ & 97,2 & 90,3 & 7,6 \\
\hline $\begin{array}{l}\text { I5. Dépót lipides (kg) } \\
\text { (engt)..... }\end{array}$ & 63,6 & 62,2 & 64,8 & 49,6 & $5^{2,2}$ & $47, \mathrm{I}$ & 54,9 & 13,7 \\
\hline 16. Dépôt protéines $\left(\mathrm{eng}^{\mathrm{t}}\right)$ & 34,4 & 32,3 & 36,9 & 37,0 & 41,8 & 47,6 & $39, \mathbf{I}$ & $5, \mathrm{I}$ \\
\hline $\begin{array}{l}\text { Dépôts (lipides } \\
\text { protéines) } \\
.\end{array}$ & 98,0 & 94,5 & Ior, 7 & 86,6 & 94,0 & 94,7 & 94,0 & \\
\hline
\end{tabular}


TABLEAU 2

Composition anatomique de la carcasse, du poids vif vide, du $5^{\mathrm{e}}$ quartier en fonction du type génétique Anatomical composition of carcass, empty body and fifth quarter according to genetic type

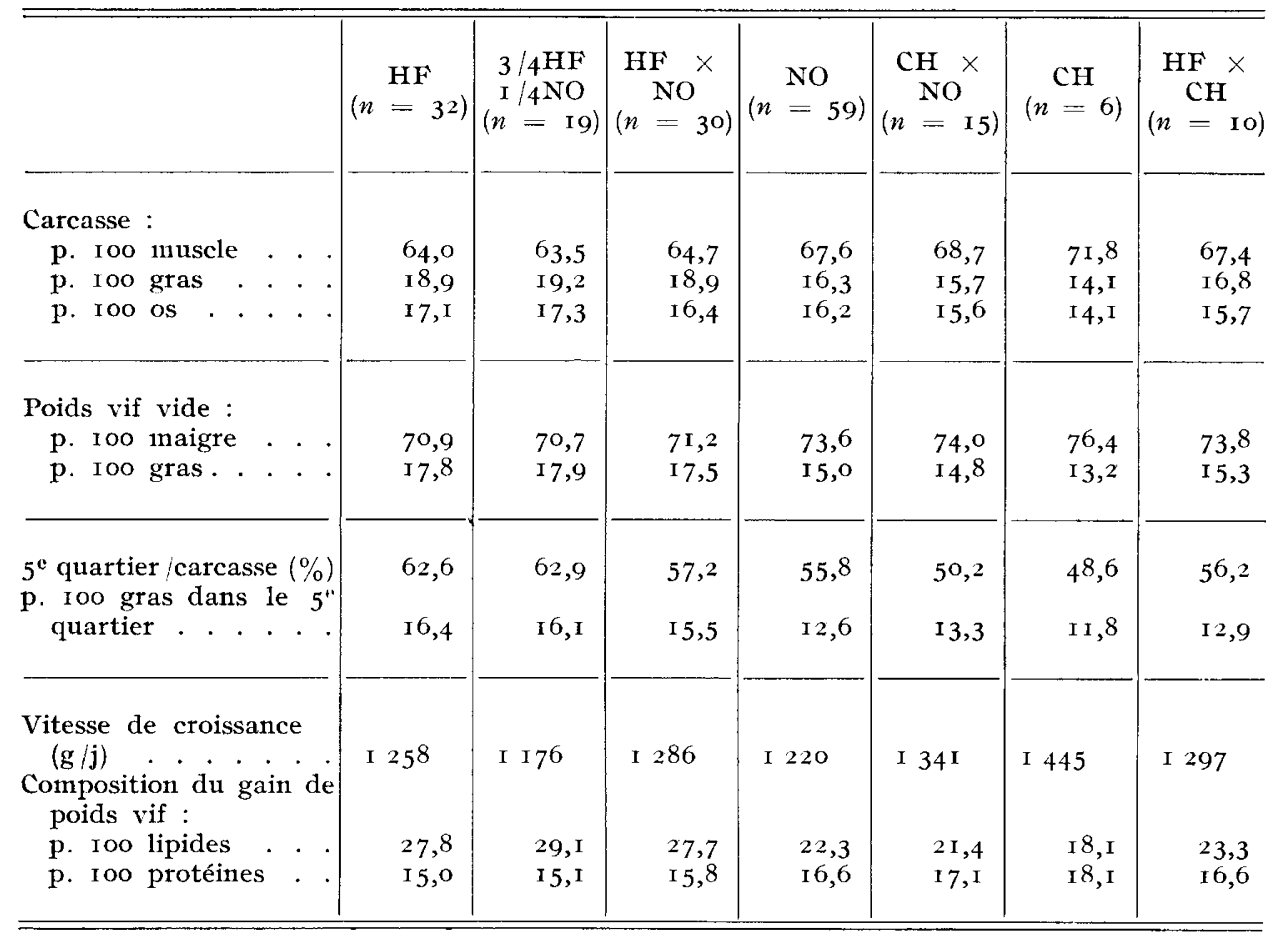

- augmentation du poids de la masse maigre et du poids de protéines à l'abattage ou déposées au cours de l'engraissement,

- diminution du poids des dépôts adipeux totaux et du poids de lipides à l'abattage ou déposés au cours de l'engraissement.

Au tableau 2 figurent la composition anatomique de la carcasse, du poids vif vide, et $\mathrm{du} 5^{\mathrm{e}}$ quartier. Les valeurs trouvées pour les races Holstein et Charolaise en ce qui concerne la composition de la carcasse sont très proches de celles trouvées par GEAY et MALTERRE (I973) sur les races Frisonne et Charolaise, respectivement: les taurillons Pie-Noirs ont en valeur relative nettement moins de muscle et plus de gras et d'os dans la carcasse que les taurillons Charolais. La race Normande se trouve pratiquement à mi-chemin entre les races Holstein et Charolaise. Les écarts de composition sont pratiquement analogues sur le poids vif vide en dépit de l'importance, très variable suivant les races, du $5^{\mathrm{e}}$ quartier par rapport à la carcasse : c'est que la composition du $5^{\mathrm{e}}$ quartier est en étroit parallèle avec la composition de la carcasse. Ces dernières constatations permettent de dire qu'une analyse intergénotypique de la consommation alimentaire en référence unique aux tissus de la carcasse aboutirait à des résultats biaisés. Les vitesses de croissance sont très variables. Il est remarquable de constater que la vitesse de croissance en race Charolaise est maximale alors que la somme des dépôts protéi- 
ques et lipidiques au cours de l'engraissement est inférieure à celle correspondant à tous les autres types génétiques, excepté du génotype Normand (tabl. I). Un tel résultat s'explique par une fixation plus importante d'eau, liée à une fixation plus importante de protéines : d'après VAN Es (I976a), la fixation d'I g de protéines s'accompagne de la fixation de $3,2 \mathrm{~g}$ d'eau. Nous retrouvons pratiquement ces valeurs puisqu'en raisonnant sur les moyennes par génotypes, nous trouvons : variation de poids vif $(\mathrm{kg})=27,5+\mathrm{I}, 03$ (lipides fixés) $+3,88$ (protéines fixées) $(r=0,99)$. L'examen des compositions du gain de poids révèle, comme il fallait s'y attendre d'après les résultats du tableau I, de spectaculaires différences entre les races pures : le gain de poids des taurillons Holsteins est plus riche en lipides et moins riche en protéines que celui des taurillons Charolais. Les valeurs obtenues sont proches de celles de Gray et al (1974).

Les animaux croisés se situent en général entre les animaux des races parentales. Cependant il existe de notables exceptions. D'une part, lecroisement Holstein $\times$ Normand s'accompagne d'efiets d'hétérosis importants sur les différents poids analysés (poids vif, poids des tissus, poids des constituants chimiques), d'autre part, on note que les animaux issus des 4 types de croisements sont systématiquement plus gras (carcasse, poids vif vide, croît quotidien) qu'on ne pouvait l'attendre d'après les performances des races parentales dans l'hypothèse d'absence d'effets d'hétérosis.

\section{b) Analyse intragénotypique sur l'ensemble des variables (modèle I)}

On constate au tableau $\mathrm{I}$ que la variation résiduelle pour la consommation est assez considérable $\left(s_{r}=0,97 \mathrm{~kg}\right.$ MS soit environ ro $\mathrm{p}$. Ioo de la moyenne générale). Le coefficient de variation résiduel pour la quantité de lipides déposés est par ailleurs nettement plus important que pour la quantité de protéines (24 p. Ioo par rapport à I3 p. I00). C'est encore le poids moyen à l'engraissement qui est le moins variable $(\mathrm{CR}=8 \mathrm{p}$. Ioo (tabl. I).

L'examen des corrélations résiduelles au tableau 3 permet de constater que la consommation est très liée au poids vif vide $(\boldsymbol{r}=0,78)$ au poids de lipides $(r=0,79)$ au poids de protéines $(r=0,60)$. Cependant la consommation étant liée au poids initial $(0,54)$, les corrélations avec les poids de lipides et de protéines déposés sont plus faibles $(r=0,76$ et 0,40 respectivement). On note par ailleurs que les deux types de dépôts ne présentent aucune corrélation entre eux $(r=0,03)$, ce qui illustre bien la variabilité individuelle pour les voies d'utilisation de l'énergie métabolisable. Le gain de poids vif pendant l'engraissement est pratiquement totalement expliqué par ces deux variables $(r=0,94)$, le coefficient de régression partielle correspondant au dépôt de lipides étant de 0,8 et celui relatif au dépôt de protéines étant de 4,4 . On note que ces résultats sont très voisins de ceux de VAN Es (valeurs attendues : I et 4,2 respectivement). Ce dernier coefficient de régression très élevé explique qu'en dépit d'une variabilité inférieure, l'importance des dépôts protéiques est beaucoup plus liée à la vitesse de croissance que l'importance des dépôts lipidiques $(r=0,85$ et 0,42 respectivement). L'analyse intraraciale confirme donc l'analyse interraciale : la vitesse de croissance dépend essentiellement du niveau de protéinogénèse.

La corrélation est pratiquement parfaite entre le poids des dépôts adipeux et le poids de lipides d'une part, le poids de la masse maigre et le poids de protéine d'autre part. On peut ainsi penser qu'intrarace la composition de la masse maigre en protéines et en lipides est pratiquement constante d'un animal à l'autre alors 


\begin{tabular}{|c|c|c|}
\hline \multirow{17}{*}{ 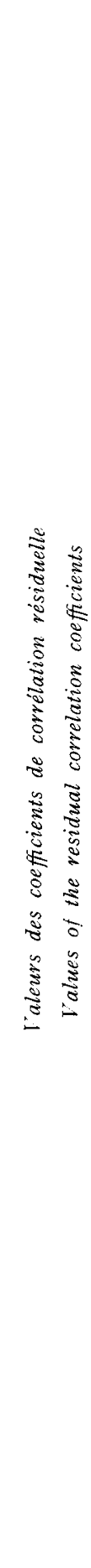 } & $\stackrel{0}{=}$ & 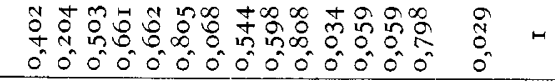 \\
\hline & $\Xi$ & 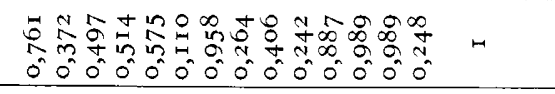 \\
\hline & $\underset{f}{E}$ & 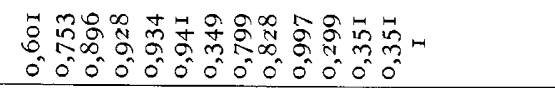 \\
\hline & $\Xi$ & 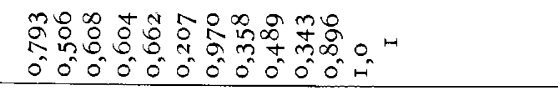 \\
\hline & $\overline{\mathbb{E}}$ & 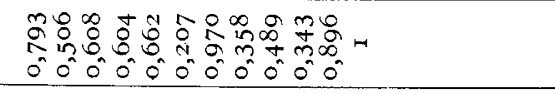 \\
\hline & $\Xi$ & 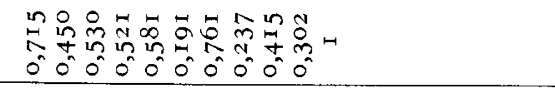 \\
\hline & 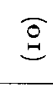 & 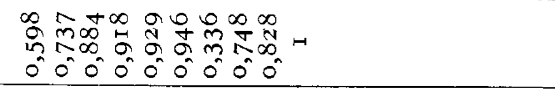 \\
\hline & a & 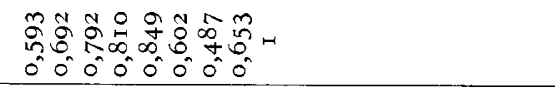 \\
\hline & $\bar{\infty}$ & 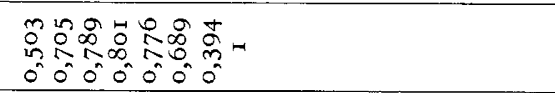 \\
\hline & $E$ & 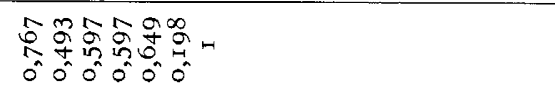 \\
\hline & $\bar{\theta}$ & 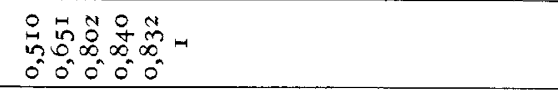 \\
\hline & $\sqrt{n}$ & 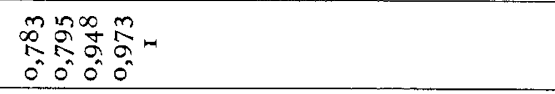 \\
\hline & ङ & 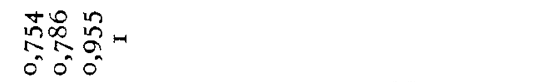 \\
\hline & $\overline{0}$ & $\begin{array}{l}n \\
m: \\
12 \\
0 \\
0\end{array}$ \\
\hline & II & $\begin{array}{c}\hat{2} \\
\text { ñ. } \\
0^{2}\end{array}$ \\
\hline & $\Xi$ & $m$ \\
\hline & & $\begin{array}{l} \\
\cdots \\
\cdot\end{array}$ \\
\hline
\end{tabular}


que ce n'est pas le cas quand on compare des races différentes (GEAY et ROBELIN, I978). Même en raisonnant intrarace, les quantités de gras et de muscle dans la carcasse ne permettent que des estimations moins bonnes des quantités de lipides et de protéines présentes dans l'animal entier $(r=0,97$ et 0,94 respectivement).

\section{c) Analyse de la consommation alimentaire}

La consommation alimentaire est liée aux besoins d'entretien appréciés à partir du poids moyen de l'animal pendant le contrôle et au dépôt d'énergie dans la masse corporelle. Nous nous efforcerons donc d'évaluer au niveau de chaque individu l'importance de ces deux sources. Cette décomposition permet ensuite de comprendre l'incidence de la vitesse de croissance et de la composition du $\mathrm{kg}$ de gain sur les variations d'efficacité alimentaire.

\section{TABLEAU 4}

Estimées de moindre carrés des effets génotypes et analyse de variance pour la consommation journalière de matière sèche $(\mathrm{kg})$

Least squares estimates of genotype effects and variance analysis for daily dry matter consumption $(\mathrm{kg})$

Analyse I : modèle sans covariable.

Analyse 2 : modèle avec 3 covariables : poids moyen, poids de lipides et poids de protéines déposés au cours de l'engraissement.

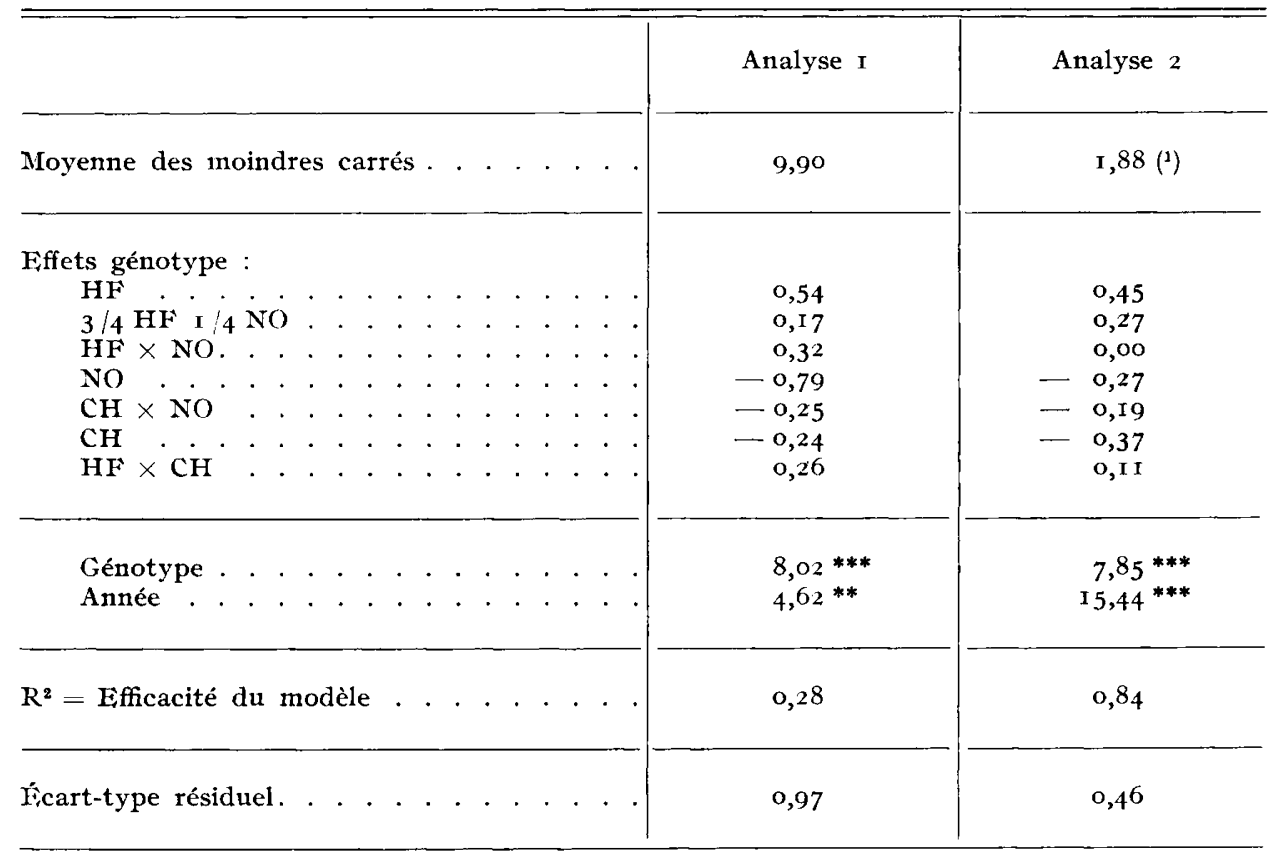

(I) Valeur obtenue quand toutes les covariables sont nulles. 
Dans notre cas particulier, le dépôt d'énergie pendant l'engraissement ne peut être apprécié que de façon indirecte. La quantité d'énergie présente dans la masse corporelle en fin d'engraissement peut être reliée au poids de lipides et de protéines obtenu sur le corps entier au moment de l'abattage. En fait, nous ne disposerons que d'estimations, faisant appel à la méthode proposée par RoBEr,IN et GEAy (1978). Le poids des lipides et des protéines présents en début d'engraissement est plus difficile à apprécier. La seule donnée de base dont on dispose à ce moment est en effet le poids vif. Les valeurs obtenues par RoBELIN et GFAY (I978) en ce qui concerne la composition en lipides et en protéines de jeunes taurillons Limousins, Salers, Salers $\times$ Charolais à 9 mois ne semblent pas montrer d'inuportantes différences de composition corporelle suivant les génotypes quand la base de référence est le poids vif. Les valeurs trouvées par Schulz et al. (I974) pour un poids de $300 \mathrm{~kg}$ au même âge, sur taurillons Frisons, ne montrent pas non plus un écart sensible par rapport à ces valeurs. Nous considérons donc dans une première approximation que la composition corporelle en début d'engraissement ne varie pas suivant le génotype et nous adoptons les valeurs de SchulTz: 8 p. roo pour la proportion de lipides et I5 p. Ioo pour la proportion de protéines, exprimées par rapport au poids vif.

Dans ces conditions, la consommation est analysée suivant un modèle de type (2), précédemment décrit, en incluant trois covariables : le poids moyen au cours de l'engraissement, les dépôts de lipides et de protéines formés au cours de l'engraissement (tabl. 4).

Les résultats de 1'analyse de variance figurent aux tableaux 4 et 5 . On remarque que le modèle (2) est nettement plus efficace que le modèle ( $\mathrm{r}$ ) puisque le coefficient de détermination $\left(R^{2}\right)$ passe de 0,28 à 0,84 , toutes les covariables ayant une in fluence significative. Les différences entre génotypes sont encore très significatives. On note que l'écart entre la race Holstein et la race Charolaise est prati-

TABLEAU 5

Coefficients de régression partielle intragénotype intra-année de la consommation totale de matière sèche pendant l'engraissement (I82 jours) et écarts-type d'échantillonnage correspondants.

Partial regression coefficients within genotype within year of total dry matter consumption during fattening ( 182 days) and corresponding standard errors

\begin{tabular}{|c|c|c|c|c|}
\hline \multirow{2}{*}{ Variable } & \multicolumn{2}{|c|}{$\begin{array}{c}\text { Coefficients de régression } \\
\text { partielle }(\hat{\lambda})\end{array}$} & \multicolumn{2}{|c|}{$s(\hat{\lambda})$} \\
\hline & $\mathrm{Kg}$ MS & Kcal EM & $\mathrm{Kg}$ MS & Kcal E,M \\
\hline Poids moyen $(\mathrm{kg})$. . . . . . . . & I, 688 & 3833 & 0,265 & 603 \\
\hline $\begin{array}{c}\text { Poids de lipides déposés pendant } \\
\text { l'engraissement }(\mathrm{kg}) .\end{array}$ & 7,606 & I 7266 & 0,579 & I 3 I 6 \\
\hline $\begin{array}{l}\text { Poids de protéines déposées pendant } \\
\text { l'engraissement }(\mathrm{kg})\end{array}$ & 7,560 & $17 \mathrm{I} 6 \mathrm{I}$ & $\mathrm{I}, 55^{8}$ & 3536 \\
\hline
\end{tabular}


quemment inchangé alors que l'écart entre la race Normande et la race Charolaise a considérablement diminué. Là encore, les consommations corrigées par régression des animaux croisés sont intermédiaires entre celles des animaux des races parentales et l'on note que les effets d'hétérosis sur les consommations observés initialement, pour les croisés Holstein $\times$ Normand notamment, disparaissent.

\section{Discussion}

\section{a) Signification des coefficients de régression partielle}

Il est intéressant de comparer ces coefficients de régression à ceux que l'on pouvait attendre compte tenu des connaissances actuelles sur les besoins d'entretien, les quantités d'énergie nécessaires aux dépôts de gras et de protéines chez les bovins. Ces besoins sont généralement exprimés en énergie métabolisable bien que le rendement de l'utilisation de cette énergie puisse varier avec le régime alimentaire et avec les individus. La variabilité individuelle joue déjà en amont, au niveau de la digestibilité de l'énergie (VERMOREL et al., I976). Or les coefficients de régression partielle dont nous nous sommes servis pour corriger la consommation au niveau des génotypes sont des coefficients interindividus intrarace. Dans la discussion qui va suivre, nous supposerons donc que les variations individuelles intragénotypiques pour la digestibilité de l'énergie et pour le rendement de l'énergie métabolisable ne sont pas en corrélation avec les covariables prises en considération dans l'analyse des consommations alimentaires.

Le coefficient de régression partielle sur le poids moyen au cours de l'engraissement peut être transformé en coefficient de régression sur le poids métabolique moyen car dans la zone $400-500 \mathrm{~kg}$ la liaison entre $\mathrm{P}$ et $\mathrm{P}^{0,75}$ est pratiquement linéaire et $\mathrm{P}^{0,75}$ augmente de $0, \mathrm{I} 63 \mathrm{~kg}^{0,75}$ quand $\mathrm{P}$ augmente de $\mathrm{I} \mathrm{kg}$. Une première estimation des besoins journaliers par unité de poids métabolique peut donc s'évaluer d'après nos résultats à $\frac{3833}{\mathrm{I} 82} \frac{\mathrm{I}}{0, \mathrm{I} 63}=\mathrm{I} 29( \pm 20) \mathrm{Kcal} \mathrm{EM} / \mathrm{kg}^{0}, 75$.

L'écart-type d'échantillonnage est assez considérable, eu égard à la modicité des effectifs et à la complexité du modèle. Cependant la valeur que nous obtenons se trouve dans la fourchette IOO-I $40 \mathrm{Kcal} \mathrm{EM} / \mathrm{kg}^{0,75}$ où se situent un grand nombre de résultats obtenus par les nutrionnistes (WEBSTER et al., I974; KIELANOwSKI, I976; Schiemann et al., I976; Thorbex et Henckel, I976; VAN Es, I976b; VERMOREL, I976).

Les valeurs proposées actuellement par les nutritionnistes sont telles que les quantités d'énergie métabolisable nécessaires à la fixation d'une quantité donnée de protéines ou de lipides sont équivalentes, l'énergie brute plus faible des protéines étant compensée par un gaspillage énergétique plus important au moment de la fixation (WEBSTER, I976a). Nos présents résultats ne vont pas à l'encontre de ces conceptions, puisque nous obtenons pratiquement les mêmes valeurs pour les coefficients de régression partielle de la consommation sur le poids de lipides déposés et sur le poids de protéines déposées. Il est nécessaire toutefois de remarquer que nos coefficients sont assez imprécis, surtout celui qui correspond au dépôt des protéines. En ce qui concerne les valeurs absolues, on dispose actuellement de données assez convergentes sur le porc et le rat (WEBSTER, I976b; FowLER et al., I976; Kiei,ANOWSKI, I976) qui les situent à environ $55 \mathrm{KJ} / \mathrm{g}$. Cependant, d'après 
Kielanowski (I976), on doit tenir compte de la moins bonne utilisation de l'énergie métabolisable par les bovins (parce que cette énergie est fournie principalement sous forme d'acides gras volatils) et les valeurs les plus probables pour les bovins se situent alors à environ $70 \mathrm{KJ} / \mathrm{g}$ de protéines ou de lipides. En transformant nos valeurs en Joules ( $\mathrm{I} C a 1=4, \mathrm{I} 8 \mathrm{~J}$ ) nous obtenons $72,2( \pm 5,5) \mathrm{KJ} / \mathrm{g}$ lipides et $7 \mathrm{I}, 7( \pm \mathrm{I} 4,8) \mathrm{KJ} / \mathrm{g}$ protéines, ce qui est en excellent accord avec ces prédictions. Il faut signaler toutefois que seule la première valeur est significativement différente des $55 \mathrm{KJ} / \mathrm{g}$ trouvés chez le rat et le porc.

\section{b) Signification des consommations corrigées par régression}

\section{- Moyenne des moindres carrés}

Cette valeur qui représente la consommation estimée avec une valeur nulle pour toutes les covariables devrait être normalement nulle. Or elle s'élève à I, $88 \mathrm{~kg} \mathrm{MS} / \mathrm{j}$, chiffre considérable qui est très significativement différent de zéro (écart-type d'échantillonnage $=0,47 \mathrm{~kg} \mathrm{MS} / \mathrm{j}$ ). Cela peut être attribué en première analyse à l'approximation linéaire que nous avons faite pour l'estimation des besoins d'entretien. Les besoins d'entretien sont proportionnels à $\mathrm{P}^{\mathbf{0}, 75}$ et non à $\mathrm{P}$. Dans une zone de variation restreinte et pour des poids élevés, l'approximation est valable sauf quand on fait varier le poids de o à $P$. En clair, avec 1'approximation linéaire, on peut évaluer correctement de faibles variations de besoins d'entretien et non les besoins eux-mêmes.

Avec l'approximation linéaire, les besoins d'entretien moyens peuvent être évalués à 4 , Io $\mathrm{kg}$ MS/J. Si l'on y ajoute les I,88 $\mathrm{kg}$ MS non " expliqués " par la régression linéaire, on obtient en réalité $5,98 \mathrm{~kg} \mathrm{MS} / \mathrm{j}$ soit $60,4 \mathrm{p}$. Ioo des besoins totaux. A partir du besoin moyen d'entretien et du poids moyen on peut estimer de nouveau le coefficient de proportionnalité au poids métabolique; il est égal à $\frac{5,98 \times 2270}{\left(44^{2}\right)^{0,75}}=\mathrm{I} 40,8( \pm 23,6) \mathrm{Kcal} \mathrm{EM} / \mathrm{kg}^{0,75}$.

Cette dernière valeur n'est pas très différente de la première valeur obtenue (I29) et cela confirme les assertions du paragraphe précédent.

\section{- Effets génotype}

Les variations entre types génétiques pour la consommation corrigée par régression peuvent être en théorie reliées à plusieurs hypothèses : différences intergénotypiques pour la digestibilité de l'énergie, pour le rendement de l'énergie métabolisable, pour l'importance des besoins d'entretien et erreurs systématiques dans l'appréciation des dépôts.

VERMOREI et al. (I976) ne trouvent pas de différences significatives entre taurillons Charolais et Frisons pour la digestibilité de l'énergie. Les mêmes auteurs ne trouvent pas non plus de différences significatives pour le rendement de l'énergie métabolisable alors que pour GARRETT (I97I), le rendement de la transformation de cette énergie en protéines et en lipides serait meilleur pour les bouvillons Hereford que pour les bouvillons Holstein. Devant le petit nombre apparent de travaux dans ce domaine, nous supposerons qu'il n'existe pas de différence entre races pour ces critères.

En ce qui concerne l'évaluation des différences raciales pour les besoins d'entretien, les travaux sont plus abondants (GARRETT, I97I; WeBsTER et al., I974; WEBSTER et al., I976; VERMOREI, I976) et indiquent que les besoins d'entre- 
tien par unité de poids métabolique des mâles de races laitières (Holstein ou Frisonne) sont plus élevés (de 5 à $\mathrm{r} 3 \mathrm{p}$. roo) que ceux des mâles de races à viande.

Compte tenu de ce qui a été dit précédemment, nous pouvons évaluer par génotype le coefficient de proportionnalité au poids métabolique d'après la relation suivante :

$$
\theta_{i}\left(\mathrm{Kcal} \mathrm{EM} / \mathrm{kg}^{0,75}\right)=\frac{\left(\mathrm{I}, 88+\alpha_{i}+\lambda_{1}\left(\overline{\mathrm{P}}_{i}-\overline{\mathrm{P}}\right)\right) 2270}{\overline{\mathrm{P}}_{i} \mathbf{0}^{0,75}}
$$

où $\alpha_{i}=$ consommation corrigée du génotype $i$ en écart à la consommation corrigée moyenne,

$\lambda_{1}=$ coefficient de régression de la consommation sur le poids moyen,

$\overline{\mathrm{P}}_{i}=$ poids moyen du génotype $i$.

On obtient alors les valeurs suivantes :

\begin{tabular}{|c|c|c|c|}
\hline $\mathrm{HF}$ & $\mathrm{I}^{2}\left(\mathrm{I} \mathrm{I}_{5}\right)$ & $\mathrm{CH} \times \mathrm{NO}$ & I36 (I04) \\
\hline $3 / 4 \mathrm{HF}$ I $/ 4 \mathrm{NO}$ & I5O (II 4$)$ & $\mathrm{CH}$ & I32 (100) \\
\hline $\mathrm{HF} \times \mathrm{NO}$ & I4I (I07) & $\mathrm{HF} \times \mathrm{CH}$ & I43 (I09) \\
\hline $\mathrm{NO}$ & I35 (I02) & & \\
\hline
\end{tabular}

On constate que les coefficients de proportionnalité varient considérablement suivant les génotypes. Le coefficient minimum est obtenu en race Charolaise (indice I00) et le coefficient maximum est obtenu en race Holstein (indice II5). Ils sont significativement différents entre eux, étant donné que les $\alpha_{i}$ sont significativement différents et que les $\overrightarrow{\mathrm{P}}_{i}$ sont relativement peu variables. Cependant, on doit noter que l'estimation de chacun d'eux est peu précise, les écarts-type d'échantillonnage sont liés essentiellement à la précision sur l'évaluation de $\mu+\lambda_{1} \overline{\mathrm{P}}$ (quantité égale à 5,98 dans notre cas) et s'élèvent à $23-24 \mathrm{Kcal} \mathrm{EM} / \mathrm{kg} \mathrm{P}^{\mathbf{0}, 75}$.

Quels peuvent être les effets des hypothèses que nous avons prises en ce qui concerne la composition corporelle en début d'engraissement? En se plaçant dans le cas extrême de la race Charolaise, on peut se demander si l'on n'a pas surestimé le pourcentage initial de lipides (c'est-à-dire sous-estimé le dépôt de lipides) et sous-estimé le pourcentage initial de protéines (c'est-à-dire surestimé le dépôt de protéines) : ces deux erreurs s'annuleraient dans une certaine mesure. Cependant pour nous placer dans une hypothèse systématiquement défavorable à nos calculs, nous supposerons que le pourcentage initial de lipides était en fait de $6 \mathrm{p}$. Ioo du poids vif et non $8 \mathrm{p}$. Ioo (cela semble être un minimum d'après les travaux de RoBEIIN et GEAY, I978), le pourcentage initial de protéines étant correctement apprécié. Dans ce cas, il est facile de calculer que la "consommation d'entretien " des animaux Charolais a été sous-estimée de $0,26 \mathrm{~kg} \mathrm{MS} / \mathrm{j}$. Ce qui amène le coefficient de proportionnalité au poids métabolique à $\mathrm{I} 38 \mathrm{Kcal} / \mathrm{j}$. On constate que l'écart est encore sensible par rapport à la race Holstein, qui se place alors à l'indice relatif iro. Les conclusions qualitatives de cette étude ne semblent donc pas dépendre des hypothèses concernant la composition corporelle initiale.

\section{c) Utilisation possible des résultats pour la sélection}

D'après les résultats précédents, il s'avère que l'énergie métabolisable fixée est proportionnelle à la somme (lipides + protéines fixés). Soit :

$$
\mathrm{C}_{\mathrm{F}}=\mathrm{C}-\mathrm{E}=\lambda(\Delta \mathrm{L}+\Delta \mathrm{P})
$$


où $\mathrm{C}=$ consommation totale (EM),

$\mathrm{E}=$ besoins d'entretien $=\theta \mathrm{P}^{0,75}$

$\Delta \mathrm{L}=$ lipides déposés,

$\Delta \mathrm{P}=$ protéines déposées,

$\lambda=$ coefficient de proportionnalité.

On constate par ailleurs que la relation de VAN Es est vérifiée autant entre races qu'intrarace :

$$
\Delta \mathrm{W} \simeq \Delta \mathrm{L}+4,2 \Delta \mathrm{P}
$$

$\Delta \mathrm{W}=$ variation de poids vif.

Le rapport $\frac{C_{F}}{\Delta W}$ ne dépend plus dans ces conditions que de $\lambda$ et du rapport $\frac{\Delta \mathrm{P}}{\Delta \mathrm{L}}=\rho$. Inversement, connaissant $\frac{\mathrm{C}_{\mathrm{F}}}{\Delta \mathrm{W}}=\mathrm{I}_{\mathrm{C}}$ indice de consommation corrigé, on peut en déduire $p$

$$
p=\frac{\lambda-I_{C}}{4,2 I_{C}-\lambda}
$$

Les coefficients $\lambda$ et $\theta$ sont encore imprécis. Mais avec le progrès futur des connaissances, il devrait être possible à long terme, dans les stations de performance-test de jeunes taureaux destinés à l'insémination artificielle (DUTERTRE, 1975), de prévoir le rapport $\frac{\text { protéines }}{\text { lipides }}$ fixé et de là avoir une idée de la composition corporelle, étant donné que l'on connaît alors la consommation $\mathrm{C}$ et la variation de poids $\Delta \mathrm{W}$.

\section{Conclusion}

L'analyse précédente a permis de montrer que la consommation alimentaire des taurillons est liée d'une part à la somme des lipides et des protéines déposés pendant l'engraissement (la quantité d'énergie métabolisable nécessaire à la fixation d'une quantité donnée de lipides ou de protéines ayant été trouvée égale), d'autre part à leur poids métabolisable par un coefficient de proportionnalité qui semble dépendre du type génétique (en particulier quand on compare type laitier spécialisé et type viande spécialisé). Les valeurs que nous obtenons sont relativement imprécises étant donné la taille de l'échantillon mais elles ne sont pas en contradiction avec les connaissances quantitatives actuelles des nutritionnistes sur les besoins énergétiques pour l'entretien, le dépôt de protéines et de lipides.

Ces constatations permettent d'analyser les causes de variation de l'efficacité alimentaire telle qu'elle est définie au niveau pratique, c'est-à-dire par la quantité d'aliment globalement nécessaire pour le gain d'r $\mathrm{kg}$ de poids vif. Au tableau 6 , figure cet indice en $\mathrm{kg} \mathrm{MS} / \mathrm{kg}$ gain pour les différents génotypes : les variations sont considérables et en particulier l'écart est de 24 p. Ioo entre les races Holstein et Charolaise. Cet indice peut être scindé en deux sous-indices, le premier correspondant à la "charge " d'entretien afférent à $\mathrm{I} \mathrm{kg}$ de gain, le second correspondant à la quantité d'aliment effectivement utilisée pour la formation de ce $\mathrm{kg}$ de gain. Ces deux sous-indices sont eux aussi variables, surtout le second, et très corrélés entre eux (tabl. 6). Les variations du second sous-indice peuvent être facilement 


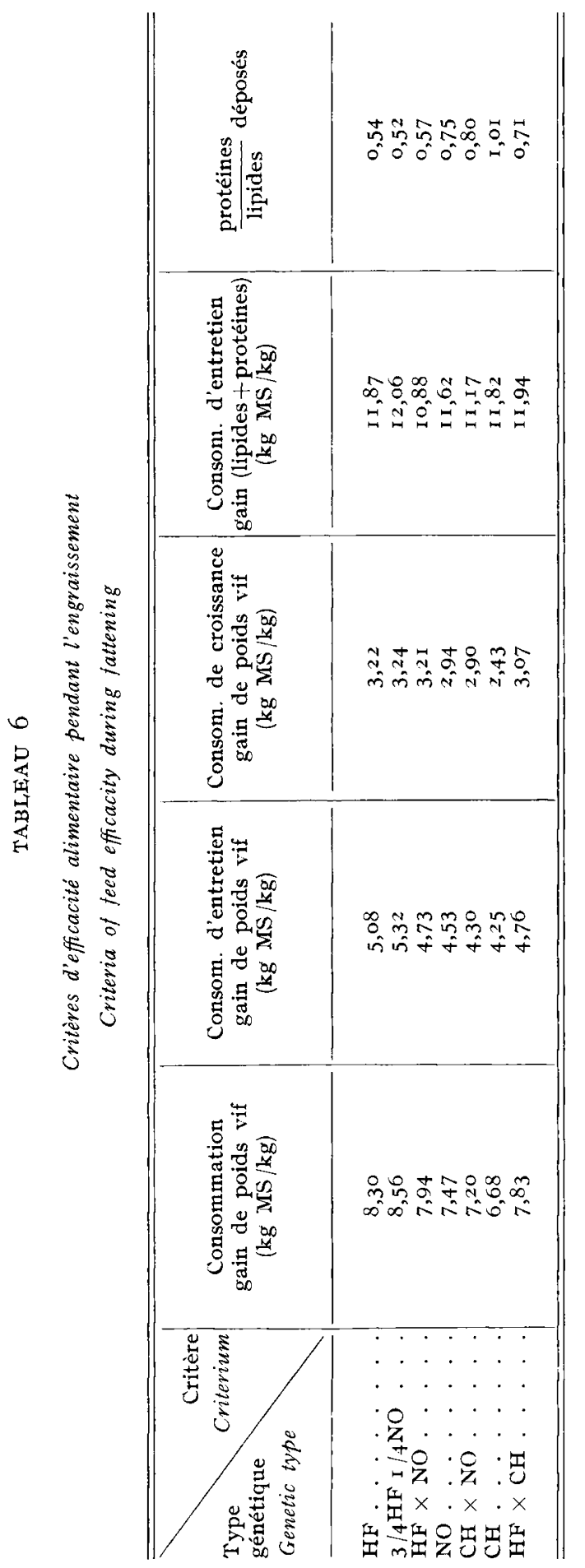




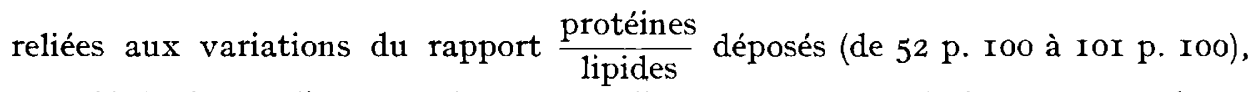
tout dépôt de protéines entraînant, nous l'avons vu, une variation correspondante du poids vif multipliée environ par 4 (grâce à une fixation d'eau). Pour le second sous-indice, la liaison avec ce rapport est moins apparente mais elle est bien réelle : si l'on rapporte la charge d'entretien non au $\mathrm{kg}$ de gain de poids vif mais au $\mathrm{kg}$ de gain de la somme (protéines + lipides), ce nouveau sous-indice est beaucoup moins variable. En particulier, les trois races pures ne diffèrent alors pratiquement pas.

Finalement, il apparaît que la cause première de la meilleure efficacité alimentaire des races à viande comparées aux races mixtes et spécialisées laitières

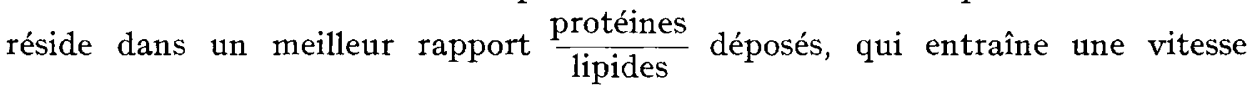
de croissance élevée et par conséquent une charge moins importante des besoins d'entretien rapportés au gain de poids vif. La seconde raison de cette supériorité des races à viande semble être aussi que les besoins d'entretien par unité de poids métabolique sont moins élevés.

Reçu pour publication en avril 1978 .

\section{Remerciements}

Nous tenons à remercier M. OLLIVIER (I.N.R.A., Jouy-en-Josas) et MM. ROlikLIN et VERMOREI. (I.N.R.A., Theix) pour leurs critiques et suggestions à la lecture du manuscrit.

\section{Summary}

\section{Some factors of variation in feed consumption of young bulls from different genetic types}

Feed consumption during fattening (from ro to i 6 months age) was measured in 17 I young bulls from 7 different genetic types : three pure breeds (Holstein, Norman, Charolais) and four various crossing between these pure breeds. The influence of "genetic type " "year of fattening", mean weight during fattening (related to maintenance requirements), amounts of lipids and proteins fixed during fattening (related to fixed energy) was analysed. Fach of these three covariables significantly affected consumption, independently of the others. The partial regression coefficient of total consumption on amount of fixed lipids was $7 x, 2( \pm 5,5) \mathrm{KJ}$ Metabolizable energy $/ g$ of lipids. That corresponding to fixed proteins was $7 \mathrm{I}, 7( \pm 14,8) \mathrm{KJ} / \mathrm{g}$. The partial regression coefficient on weight also is significant; however a simultaneous adjustment of consumptions for each covariable showed that significant differences still existed between the genetic order to explain this result, the heterogeneity of partial regression coefficients on weight was assumed, the coefficients being higher for dairy type (Holstein) than for meat type (Charolais). The coefficient of proportionality to metabolic weight $\left(\mathrm{P}^{\mathbf{0}, 75}\right)$ was $\mathrm{I} 4 \mathrm{I}( \pm 24) \mathrm{Kcal} \mathrm{ME} / \mathrm{Kg}^{0,75}$ in the whole sample. It would be $\mathrm{I}_{2} 2$ for young Holstein bulls and $\mathbf{1}^{2}$ for young Charolais bulls.

The intergenotypic variations for feed efficiency (kg DM consumed per $\mathrm{kg}$ liveweight gain) seem to be primarily related to the ratio proteins/lipids fixed during fattening, which varies from 0,54 for the Holstein breed to $\mathrm{I}, \mathrm{O}$ I for the Charolais breed. 


\section{Références bibliographiques}

COLLEAU J. J., I974. Comparaison entre la race mixte Normande, les races spécialisées Holstein Canadienne et Charolaise et leurs croisements. I-Performances de croissance des mâles et des femelles. Ann. Génét. Sél. anim., 6, 445-462.

COLLEAU J. J., I975. Comparaison entre la race mixte Normande, les races spécialisées Holstein Canadienne et Charolaise et leurs croisements. II-Performances d'engraissement et de carcasse des mâles. Ann. Génét. Sél. anim., 7, 35-48.

Demarquiliy C., ANDrieu J. et Sauvant D., i978. I.N.R.A. Alimentation des Ruminants Chap. I6: Composition et valeur nutritive des aliments. Chap. I7 : Tableaux de la valeur nutritive des aliments.

DUTERTRE M., I975. Modalités d'application, bilan et analyse du contrôle individuel des taureaux utilisés en insémination artificielle. Mémoire de fin d'études ISA de Beauvais.

Fowl.er V. R., Bichard M., Pease A., I976. Objectives in pig breeding. Anim. Prod., 21, $365-387$.

Garretit W. N., I97I. Finergetic efficiency of beef and dairy steers. J. Anim. Sci., 32, 45I456.

GeAy Y., BeRANGer C., I969. Estimation de la composition de la carcasse de jeunes bovins à partir de la composition d'un morceau monocostal au niveau de la i i côte. Ann. Zootech., 18, $65-77$.

GeAY Y., Malterre C., I973. Croissance, rendement et composition des carcasses de jeunes bovins de différentes races. Bull. tech., $C R Z V, 14$, I 7-2o.

GEAy Y., ROBELIN J., JARRIGE R., I974. Variations des quantités d'énergie, de protéines et de lipides fixés dans la carcasse par les jeunes taureaux à l'engrais. VIe Symposium sur le Métabolisme Énergétique des Animaux Domestiques (Stuttgart) EAAP Publ., 14, I39-I 42.

Jarrige R., Beranger C., Geay Y., Grenet N., Malterre C., Robei,in J., i97i. Besoins énergétiques des jeunes bovins. SEI, Étude, no ${ }_{46} 6,167-184$.

KIELANOWSKI J., I976. Introduction to the discussion on the utilization of energy and protein by growing animals. Communication at the E.E.C. Colloquium on "Feeding efficiency and interaction between genotype and nutrition in growing animals ". Theix-France.

Robeirin J., GeAy Y., 1975. Estimation de la composition des carcasses de jeunes bovins à partir de la composition d'un morceau monocostal prélevé au niveau de la i I e côte. I- Composition anatomique de la carcasse. Ann. Zootechn., 24, 391-402.

ROBELIN J., GEAY Y., I978. Estimation de la composition du corps entier des bovins. Ann. Zoot., 27 (sous presse).

Schulz E., Oslage H. J., DaEnicke R., I974. Untersuchungen über die Zusammensetzung der Körpersubstanz sowie den Stoff und Energie ansatz bei waschsenden Mastbullen. Fortschritte in der Tierphysiologie und Tierernährung. Heft 4 .

THORBEK G., HENCKel, S., 1976. Studies on energy requirement for maintenance in farm animals. Proc. 7 Symp. energy met., I I 7-I 20.

VAN Es A. J. H., I $976 a$. The efficiency of meat production on fattened animals. In " Meat animals: Growth and Productivity "Plenum Press.

VAN Es A. J. H., I976b. The utilisation of energy and nitrogen by growing animals. Communication at the E.E.C. Colloquium on "Feeding efficiency and interaction between genotype and nutrition in growing animals $n$. Theix-France.

VERMOREL, M., BoUvier J. C., GEAy Y., I976. The effect of the genotype (Normal and double muscle Charolais and Friesian) on energy utilization by growing cattle at 2 and I 6 months of age. Proc. 7 symp. energy met., 21 7-220.

Webster A. J. F., Brockway J. M., SMITh J. S., 1974. Prediction of the energy requirements for growth in beef cattle. I-The irrelevance of fasting metabolism. Anim. Prod., 19, I $27-139$.

WEBSTER A. J. F., I976a. Efficiencies of energy utilization during growth. In " Meat animals Growth and Productivity "Plenum Press, 89-102.

WEBSTER A. J. F., I976b. Communication at the E.E.C. Colloquium on "Feeding efficiency and interaction between genotype and nutrition in growing animals ". Theix-France. 